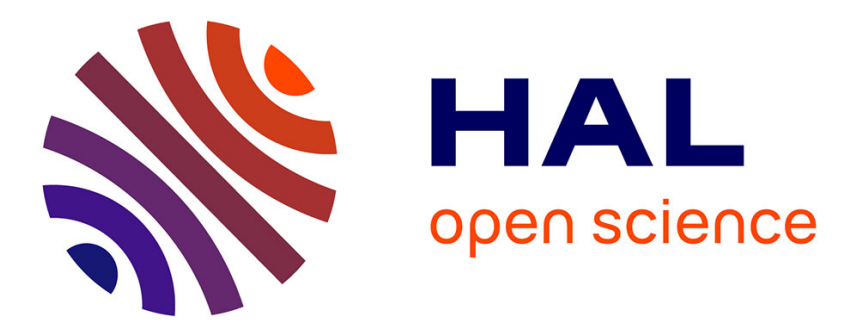

\title{
Serious Games: Is Your User Playing or Hunting?
}

Sofya Baskin, Sharon Anavi-Goffer, Anna Zamansky

\section{To cite this version:}

Sofya Baskin, Sharon Anavi-Goffer, Anna Zamansky. Serious Games: Is Your User Playing or Hunting?. 14th International Conference on Entertainment Computing (ICEC), Sep 2015, Trondheim, Norway. pp.475-481, 10.1007/978-3-319-24589-8_43 . hal-01758419

\section{HAL Id: hal-01758419 https://hal.inria.fr/hal-01758419}

Submitted on 4 Apr 2018

HAL is a multi-disciplinary open access archive for the deposit and dissemination of scientific research documents, whether they are published or not. The documents may come from teaching and research institutions in France or abroad, or from public or private research centers.
L'archive ouverte pluridisciplinaire HAL, est destinée au dépôt et à la diffusion de documents scientifiques de niveau recherche, publiés ou non, émanant des établissements d'enseignement et de recherche français ou étrangers, des laboratoires publics ou privés. 


\title{
Serious Games: Is Your User Playing or Hunting?
}

\author{
Sofya Baskin ${ }^{1}$, Sharon Anavi-Goffer ${ }^{1}$, Anna Zamansky² \\ ${ }^{1}$ Ariel University, Department of Behavioral Sciences \\ Ariel, Israel \\ ${ }^{2}$ University of Haifa, Information Systems Department \\ Haifa, Israel
}

\begin{abstract}
There is an increasing demand for entertainment applications developed for pets, in particular for dogs and cats. However, play interaction between animals and technological devices still remains an uncharted territory both for animal behavior and entertainment computing scientific communities. While there is a lot of anecdotal evidence of pets playing digital games, the nature of animal-computer play interactions is still not understood. In this paper we report on empirical findings based on observing and analyzing dog-tablet game interactions. Using categories emerging from our data analysis, we construct an ethogram, a "catalogue" of behavioral patterns typical of dog-tablet interactions. Based on our data analysis, we hypothesize that the nature of the observed interactions is that of predatory behavior, in response to stimuli in the form of "prey-like" virtual objects displayed on the screen. Based on our hypothesis, we further propose some questions for future investigation, and raise some issues that need to be addressed by game developers when targeting dogs as their users.
\end{abstract}

\section{Introduction}

The emerging discipline of animal-computer interaction (ACI) [1] aims to understand the interaction between animals and computing technology within the contexts in which the animals habitually live. Interactions between dogs and technology have been studied mainly in the context of working and assistance dogs, trained to issue alerts (on upcoming seizures due to epilepsy or low blood sugar in diabetic patients), e.g., by pulling on a string 1 or pushing a button on a multi-touch screen [3]. But in these scenarios dogs interact with a device mainly because they are trained to do so. While digital game playing for the purpose of entertainment has been explored for other animals, such as apes [4], cats [5], and pigs [6], dog users remain underrepresented in this context. And yet dogs playing tablets is a wide-spreading phenomenon that seems to be here to stay. Hundreds of mobile applications have hit the market; over 10,000 YouTube videos can be found using the keywords "dog playing tablet". In the last years several dog training centers were reported to open classes teaching dogs to use mobile devices $([7,8])$.

How should we design digital games for pets, in which they find some recreational or other positive value? So far these questions have mainly been addressed from ethi- 
cal [9] and philosophical perspectives [5], in some sense in a top-down way, deriving game design principles from more general theoretical principles. In this paper we propose to take a bottom-up approach ${ }^{1}$ by asking what can we learn about digital game design by observing what dogs actually do when playing digital games. To the best of our knowledge, behavioral characteristics of pet interactions with tablets have not been systematically investigated. These characteristics, however, are key to answering questions such as what constitutes an animal-computer play interaction, what it means for an animal to be engaged by a digital game, and how it perceives virtual objects. Exploring these questions calls for applying research methodologies from ethology.

Ethology is the comparative study of animal behavior, studying the biological roots and meanings of animal actions. One of the key steps in this process is constructing ethograms, which are quantitative descriptions of an animal's normal behavior. According to [11], "It is absolutely fundamental to any study of animal behavior to define what behavior types are being observed and recorded, and therefore production of an ethogram is always the first step in any animal behavior research". In this paper we take such a step by constructing an ethogram of typical behaviors dogs exhibit while playing digital games, based on analyzing video data of dog-tablet interactions.

\section{The Empirical Study}

Method. The first stage of the study included a careful selection of 32 videos of dogs playing digital games on tablet/iPad, according to the following selection criteria: (i) duration of each video: at least 25 seconds; (ii) minimal human intervention in the video fragment; (iii) dogs of small and medium breeds.

The main source for the video footage was YouTube. The benefits and limitations of using YouTube data for studying animal behavior have been extensively discussed in [12]. This methodology is based on the premise that the probability of capturing any given behavior is dramatically increased when the number of people obtaining the footage is not restricted to academics but is widened to the public. This is of course particularly true for the case of dog-tablet interaction: while a large variety of dogs is usually not easily available for academic studies, dog owners' videos of their pets are easily available. Nevertheless, there are limitations in terms of the use of such videos: they may not be chronologically or sequentially correct, they may be edited or even fake. For this reason we excluded from our analysis videos which included any type of editing, such as cutting scenes, speed modification, etc.

Results. An ethogram is a list of behaviors performed by particular individuals in a particular environment and for a particular purpose. Ethograms for the same species may differ depending on the individuals, environment and, most of all, the purpose of the research [11]. For constructing an ethogram, each video was tagged with behavioural patterns observed in it. The patterns were divided into the following three types:

\footnotetext{
${ }^{1}$ In a popular blog article [10] a similar approach was taken by looking at YouTube videos of dogs and cats playing iPad games. Their focus was however on the question whether dogs or cats are "better iPad players" (cats scored a higher proficiency). No description of the data collection and analysis methodologies was provided.
} 
Attention to tablet: Activities expressing an interest towards physical aspects of the tablet without getting into physical contact with it. This includes eye-tracking the virtual object and head twisting.

Actions physically directed at tablet: Activities based on a physical contact with tablet or moving around it, including scratching the tablet screen, catching the virtual object by teeth, pushing the tablet, licking the tablet, jumping around the tablet with attention to virtual object or performing fox jump. Fox jump (also known as a stiff legged jump) is a typically predatory movement, the dog rearing up on his hind legs and slamming both straightened and held rigid front legs down to the ground. The dog throws significant weight onto the front legs creating a great deal of downward force. Sometimes dogs do fox jumps in predatory games with small balls.

Actions not directed at tablet: Other activities not directly targeting the tablet, including heavy panting, nose licking, nervous yawn and catching the owner's hands.

The observed behavioral patterns together with their classification are summarized in Table 1 below. Their occurrence percentage (out of 32 videos) appears in brackets.

\begin{tabular}{|l|l|l|l|}
\hline Category & Attention to tablet & Physically directed at tablet & $\begin{array}{l}\text { Not directed at } \\
\text { tablet }\end{array}$ \\
\hline & $\begin{array}{l}\text { Eye-tracking the object } \\
(87 \%)\end{array}$ & $\begin{array}{l}\text { Scratching the screen (90\%) } \\
\text { Catching object by teeth (56\%) }\end{array}$ & $\begin{array}{l}\text { Heavy panting } \\
(16 \%)\end{array}$ \\
& Head twisting (9\%) & $\begin{array}{l}\text { Trying to catch } \\
\text { owner's hand } \\
(15 \%)\end{array}$ \\
Behavior & & Licking the tablet (25\%) & $\begin{array}{l}\text { Nose licking } \\
(9 \%)\end{array}$ \\
& & $\begin{array}{l}\text { Fox jump (12,5\%) } \\
\text { Pushing tablet by nose (9\%) } \\
\text { Jumping around the tablet with } \\
\text { attention to object (3\%) }\end{array}$ & $\begin{array}{l}\text { Nervous yawn } \\
(3 \%)\end{array}$ \\
\hline
\end{tabular}

Table 1: Behavioral patterns classified according to basic types of behaviors

The distribution of game types was as follows (the number in brackets is the number of videos out of 32): fish catching (14), Fruit Ninja (10), running rat (4), running laser pointer (3), volleyball (1).

\section{Discussion}

\section{Predatory play or serious hunting?}

All the observed behavioral patterns obtained in our ethogram directed at the tablet seem to be different types of either predatory behavior or predatory play. Predation is the act of an individual or group of individuals of one species (the predator) consuming an animal of another species (the prey). Predation (or hunting) consists series of stages: encounter, detection, recognition, attack, capture and consumption. Predatory 
play is a seemingly aimless manipulation of objects or rapid movements, but in fact may be similar to the first stages of predation without consumption.

The observed actions in the first two ethogram categories, attention to tablet and physically directed at tablet, may all be classified as different types of encounter, detection, recognition, attack, capture, all of which are components of both serious hunting and predatory play. Moreover, none of the dogs exhibit key social play postures such as play bow (the front-end-lowered rear-end-up position of play intention posture) and raised forepaw, reinforcing our belief that we are witnessing predatory behavior (either serious or playful). More concretely, the behavioral patterns we witnessed may be classified as fixed action patterns (FAPs) of predatory behavior, rather than social play behavior. FAPs are predictable, genetically predetermined and rigid sequences of behavior, triggered by simple stimuli called sign stimuli and resulting in simple responses [13]. Once triggered, these responses are unchangeable and must be carried to completion. In our context the sign stimuli are the virtual objects which have some characteristics of natural prey, such as size and mobility. Due to the fact that FAPs are genetically encoded reflex-like actions, the behavioral patterns in dog-tablet interactions with prey-like digital games are expected to be highly predictable, leaving almost no expressive freedom to the dog.

While all participants exhibited predatory behavior patterns, it is likely that their intensity varies depending to dog breed. In most breeds, the intensity of the predatory response has also been significantly reduced, while in others certain fragments of predatory patterns have been reinforced through selective breeding [14]. While in most breeds, the intensity of the predatory response has been significantly reduced, hunting breeds, save most of behavioral activities and, an opposite, some of predatory behaviors were increased [15]. Terrier breeds have a strong predatory response that is directed toward small mammals, and are therefore expected to have the strongest reaction to prey-like digital game. It is interesting to explore the dependency of the behavioral patterns exhibited by participants on their breed.

We do not have enough data to make a clear distinction between predatory play and actual hunting. Moreover, the same dog may start out the interaction in predatory play, but end up hunting seriously (as we believe was the case in several videos). During this process, its frustration from not being able to perform the consummatory act may grow, as described next.

Digital games - a source of frustration?

The third type of actions (not directed at tablet) deserves special attention. We believe that these actions may be signs of nervousness and frustration which take the following two forms:

Displacement activity: Displacement (ambivalent) behavior occurs when an animal is in some kind of a motivational conflict between two or more tendencies. If such conflict remains unresolved, this is likely to result in a state of frustration and nervousness. Signs of such behavior are nose licking (generally considered a submissive behavior, showing ambivalence) and yawning, (if performed not during rest, it is also usually interpreted as displacement activity in ambivalent situations) [11].

Redirected aggression: Aggressive behavior may be redirected towards other targets if the ones engaged in the confrontation are unreachable. The difference between this 
type of behavior and displacement activity is that in the former the target for the behavior is substituted, rather than the behavior itself. In our observations, redirected aggressions was expressed in situations where dogs playing tablet switched their attention to the hands of their owner during their interaction. Thus due to their inability to catch the virtual object, they redirected their aggression towards real objects. This also indicates that aggressive dogs playing with a tablet may be dangerous to their surroundings.

These types of behavior may be an indication of a significant motivational conflict caused by predatory motivation together with the inability to commit the final consummatory act (catching the virtual object), which leads to frustration and stress of the dog. This leads to the question which individual characteristics of participants, games or contexts are more likely to induce stress instead of enjoyment in game interactions.

\section{Fruit ninja, running rat...or something completely different?}

Predatory FAPs are triggered by sign stimuli, which is usually a subset of sensory information. Humans use a much richer set of sensory information than animals: e.g., observing that our virtual object has ears and nose of a particular form and has grey color, we classify it as a rat. However, dogs seem to have their own different subset of associations: e.g., they do not seem to make a clear distinction between a rat and a laser as the virtual moving object. Yet, they may distinguish between these objects and fish or Fruit Ninja objects: catching objects with their teeth mostly occurred in fruit ninja ( 7 out of 17) and fish catching (5 out of 17). Interestingly, while they exhibited a stronger predatory reaction to Fruit Ninja objects, fox jumps, strongly associated with predatory behavior, occurred only in animal-like objects (fish and rat). This leads to the question how behavioral characteristics of virtual objects are related to the different types of predatory behaviors.

\section{Summary and Future Research}

Videos of pets playing iPad games going viral on social networks, as well as an increasing number of applications with pets as their target audience call for a deeper understanding of animals' behaviors and perceptions in these interactions, as well as of the benefits and potential dangers of digital game playing for animals. While animal-tablet interactions have been explored for cats, apes and pigs, to the best of our knowledge, this is the first study which addresses dog behavior in this context.

In this paper we have presented a preliminary ethogram of dog behavior patterns during interactions with digital games. It unveils the complexity and ambiguity of dogtablet interactions, showing a variety of predatory behavior patterns, ranging over exploration, physical acts directed at the tablet, signs of frustration and redirected aggression.

It is evident that dogs are easily attracted to digital games. In light of our results, one needs to take into account that some dogs may find digital games stressful. Nevertheless it seems that playing digital games has a potential of improving the dogs' welfare: by cognitive stimulation and enrichment, stress reduction, physical exercise via typical 
game movements, etc. We plan to further investigate this aspect, using non-invasive methods, such as cortisol level in saliva and heart rate measurements.

The limitations of our study need to be taken into account. An important issue is the use of YouTube videos, in light of the fact that we have no control over the conditions in which the video footage was taken. While this may be acceptable for producing a preliminary ethogram, further data needs to be collected in controlled experiments to validate the results.

\section{References}

1. Mancini, C. Animal-computer interaction: a manifesto. Interactions 18.4 (2011): 6973.

2. Robinson, C. L., Mancini, C., van der Linden, J., Guest, C., \& Harris, R. Caninecentered interface design: supporting the work of diabetes alert dogs. The $32 \mathrm{nd}$ annual ACM conference on Human factors in computing systems, 3757-3766, 2014.

3. Zeagler C., Gilliland S., Freil L., Starner T., and Jackson M..Going to the Dogs: Towards an Interactive Touchscreen Interface for Working Dogs In UIST ACM Symposium on User Interface Software and Technology Honolulu, HI, USA 2014.

4. Boostrom, H. Problem-Solving with Orangutans (Pongo pygmaeus and Pongo abelii) and Chimpanzees (Pan troglodytes): Using the IPAD to Provide Novel Enrichment Opportunities. Diss. Texas A\&M University, 2013.

5. Westerlaken, M., and S. Gualeni. "Digitally complemented zoomorphism: a theoretical foundation for human-animal interaction designThe 6th International Conference on Designing Pleasurable Products and Interfaces. ACM, 2013.

6. Playing with pigs, http://www.wired.co.uk/news/archive/2013-03/11/human-animalgaming

7. iPad classes for dogs http://www.theregister.co.uk/2013/08/28/ipad_classes_for_dogs_offered_in_new_yor $\mathrm{k} /$

8. iPad classes for dogs, http://www.padgadget.com/2014/03/21/idog-clinics-use-ipadto-teach-dogs-to-read/

9. Väätäjä, H. K., and E.K. Pesonen. Ethical issues and guidelines when conducting HCI studies with animals. CHI'13 Extended Abstracts on Human Factors in Computing Systems. ACM, 2013.

10. Analyzing cat and dog tablet interactions, http://www.bigfishgames.com/blog/chewon-this-analyzing-200-dogs-cats-playing-with-ipads/.

11. Mills, D.S., and Marchant-Forde J.N., eds. The encyclopedia of applied animal behaviour and welfare. CABI, 2010.

12. Nelson, X.J., and N. Fijn. "The use of visual media as a tool for investigating animal behaviour." Animal Behaviour 85.3 (2013): 525-536.

13. Gadbois, S., Sievert, O., Reeve, C. , Harrington, F. H, Fentress, J.C.,"Revisiting the concept of behavior patterns in animal behavior with an example from food-caching sequences in Wolves (Canis lupus), Coyotes (Canis latrans), and Red Foxes (Vulpes vulpes)." Behavioural processes 110 (2015): 3-14.

14. Fox, M.W., "The Dog: Its Domestication and Behavior". Garland Press, New York, 1978.

15. Scott, J. P., ed. "Genetics and the Social Behavior of the Dog". University of Chicago Press, 1965. 